\title{
MOBILE and the provision of total joint replacement
}

\author{
Paul Dieppe, Diane Dixon, Jeremy Horwood, Beth Pollard, Marie Johnston on behalf \\ of the MOBILE research team \\ Department of Social Medicine, University of Bristol, Bristol, UK
}

Modern joint replacements have been available for 45 years, but we still do not have clear indications for these interventions, and we do not know how to optimize the outcome for patients who agree to have them done. The MOBILE programme has been investigating these issues in relation to primary total hip and knee joint replacements, using mixed methods research.

There have been five main strands:

(1) Epidemiological investigations to find out who is receiving total hip and knee replacements in the National Health Service (NHS). This has shown that there are extensive variations in different regions of the UK, with inequalities and probable inequities in the provision of these operations;

(2) Epidemiological work to ascertain the population-based needs for the operations, showing under-provision of knee joint replacements, and a relative reluctance of both patients and GPs to consider knee surgery;

(3) Quantitative and qualitative research into the views of patients, health care professionals and the public on the indications for, and prioritization of, total hip and knee joint replacements. This has shown lack of agreement within or between professional groups, as well as a mismatch between the views of patients and the public, and those of professionals;

(4) Theoretical and experimental work on patient-related outcome measures, and the development of new instruments to assess both pain and function in people with osteoarthritis, based on the International Classification of Function, as well as a new integrated model of function;

(5) Cohort studies of patients undergoing hip or knee joint replacements to find out what the determinants of good and bad outcomes are. These studies have emphasized the huge variation in disease severity at the time of surgery.

The challenge now is to use and implement our findings for maximum patient benefit.

\section{Introduction: what questions does this programme address?}

One of the first research programmes to be core funded within the MRC HSRC was on the provision of total joint replacements (TJR). The work was approved by MRC in 1999 and initially led by a multidisciplinary team drawn from the collaboration, including Linda Davies (health economics), Paul Dieppe (rheumatology and health services research), Jenny Donovan (social science), Matthias Egger (epidemiology and statistics), Paul Gregg (orthopaedic surgery) and Marie Johnston (health psychology).

Initially we set out to answer two main questions:

(1) What should the indications for a total joint replacement be in someone with osteoarthritis of the hip and/or knee?

(2) How can we optimize the outcomes for people with osteoarthritis who undergo a total joint replacement?

Although high quality clinical trials are difficult to do in this area, we realized that such trials might be needed to provide definitive answers to the questions posed; therefore, our first programme was entitled 'Towards Trials of Total Joint Replacement'. Subsequently it became clear that our questions were inextricably linked to an understanding of musculoskeletal pain and disability 
in older people, so in the second quinquennium we combined the total joint replacement work with Shah Ebrahim's programme on mobility in the over-65s to form the MOBILE research project, led by Paul Dieppe, Shah Ebrahim and Marie Johnston.

\section{What was known about this subject 10 years ago?}

Modern joint replacement surgery was introduced by the pioneering hip prosthesis work of Sir John Charnley in the $1960 \mathrm{~s}$. ${ }^{1}$ It was immediately clear that these hip replacements could revolutionize the lives of people with severe arthritis, removing their pain and restoring mobility. However, although Charnley's overall results were good, it soon became apparent that infections and prosthesis loosening could also be major problems following joint replacement. The subsequent introduction of knee replacements, which initially had relatively poor outcomes, resulted in the technical issues of prosthesis design, bone fixation, early and late loosening, and sepsis coming to the top of the research agenda. Prosthesis designs (particularly for the knee) soon improved, and so did outcomes. Although trials of TJR were never undertaken, the results of cohort studies, along with the testimonies of countless patients and health care professionals, led to the widespread belief that total hip or knee joint replacements are safe, effective procedures. $^{2}$

However, the surgeons remained preoccupied with the technical issues of prosthesis design, surgical procedures, prosthesis loosening and infection, rather than patient outcomes. Furthermore, the patient outcome measures being used most often in surgical practice, such as the American Knee Society scoring system or Harris Hip Score ${ }^{3,4}$ had not been properly validated, and they mixed different types of measure, summing scores that came from patient report and physician examination, for example.

The demand for hip and knee replacements increased greatly in the 1980s and 1990s, resulting in extensive waiting times for patients. In spite of 'political' concern about this, surprisingly little research work was undertaken on the indications for TJR or on patient outcomes. Some consensus statements, based largely on the views of surgeons, were published $;^{5}$ in Canada and New Zealand more formal attempts to aid patient prioritization were undertaken; ${ }^{6,7}$ and in the USA one of the 'PORT' (patient outcomes research teams) studies addressed knee replacement. ${ }^{8}$ In Scandinavian countries, joint replacement registries were set up, largely to track the numbers of procedures being undertaken in different units and to relate late loosening (or prosthesis failure) to the design of the prostheses. ${ }^{9,10}$ But the research field was, and still is, heavily dominated by the work of the materials scientists and engineers who, along with the surgeons, are continually trying to improve the design and longevity of their prostheses.

So, at the end of the 20th century, when we began our work, TJR was already a very high volume, expensive,

high profile medical intervention throughout the developed world. It was known that some $80 \%$ of these procedures were done for people with osteoarthritis (OA), and that most, but not all patients were pleased with the results. However, there were big gaps in our understanding of who was getting a TJR and why, or who should be offered these interventions, and how good patient outcomes after hip or knee replacement really were. We knew very little about how we should be assessing outcome, and patients' perspectives had not been investigated. Furthermore, research was not being undertaken in the UK on the process of care relating to TJR, or on how the experience and outcomes for people undergoing these operations might be improved.

Therefore, when the HSRC was set up, primary joint replacement for OA of the hip or knee seemed to be an ideal context for multidisciplinary health services research, and one that might provide generalizable insights into key factors determining the 'effectiveness, efficiency and experience ${ }^{, 11}$ of major health care interventions within the NHS.

\section{What has the programme achieved?}

We began with literature reviews and a theoretical consideration of the pathways to joint replacement in the $\mathrm{UK}$, including the factors that might affect indications and utilization. ${ }^{12}$ We have based much of our thinking and work around this framework (Figure 1), and it led to the development of four main strands within our subsequent work:

\section{THE PATHWAY}

People in the community with $\mathrm{OA}$



The 'gatekeeper'

(usually the GP in the UK)

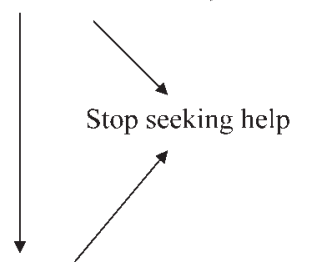

The orthopaedic surgeon



Figure 1 The patient pathway

$$
\text { Surgery }
$$

Perceptions about self and disease Previous health care experiences Experiences of others they know

Negative experience with doctor Different perceptions of severity Medical concerns on fitness

\section{Not considered 'suitable'} Not thought to be 'motivated' 
(1) Epidemiological work asking two main questions:

- Who is the NHS providing TJRs for at present, and what trends are there in provision?

- What are the population-based needs for TJRs in England?

(2) Quantitative and qualitative work to investigate the views of patients, professionals and the public on their experiences of services, on indications for and prioritization of joint replacement, and more recently on the experience of waiting for a TJR;

(3) Theoretical and practical work on patient-related outcomes, and the measures being used to assess clinical outcomes after TJR, leading to the development and validation of new instruments and further theoretical developments in the disability field;

(4) Cohort studies of patients undergoing TJRs, designed to help study their outcomes and uncover the determinants of relatively good or bad results.

However, one of our main aims has been to develop a genuinely multidisciplinary research programme in which the different disciplines and projects contribute to answering the questions posed. So a key issue for the research team has been finding ways to bring these different strands of work, dominated by different disciplines, together to further equitable, effective and efficient provision of TJRs in the NHS.

\section{Epidemiology}

\section{The provision of TJRs in the English NHS}

We used the Hospital Episode Statistics (HES) database to investigate current and likely future provision of TJRs in England, and to investigate some aspects of equity in provision and determinants of bad outcomes.

The 'headline' findings were: ${ }^{13-15}$

- that rates of provision of TJR in England are continuing to rise, particularly for primary knee joint replacements and revision hip joint surgery. Knee joint replacement will soon become a higher volume intervention than hip replacement (the most recent HES data shows this has now happened);

- more older people are receiving joint replacements than was the case 10 years ago;

- age/sex standardized rates of surgery vary by 25$30 \%$ between the different health regions of England, with marked differences in sex ratios in different areas, raising concerns about possible inequities and inequalities in provision;

- adverse outcomes are less likely to occur in high volume surgical units and in units that do surgical training, suggesting the need for more standardization of procedures and centralization of services in England.

\section{Population-based needs assessments}

Coverage of the 'political' aspects of TJR, referred to above, sometimes implies that there is a bottomless pit of need, and that services could never cope with the numbers of people who might benefit from such interventions. This is obviously nonsense, but there had been almost no empirical work done on populationbased needs for these procedures in the UK, and those who are responsible for delivering services need such information. We used the Somerset and Avon Survey of Health data-set (SASH) to investigate this. ${ }^{16,17}$ The SASH cohort is a cross-sectional survey of a stratified (according to the UK population) random sample of 28,080 individuals aged 35 years and over; from the data obtained in this cohort we estimated the requirement for joint replacement on the basis of levels of pain and activity ability (based on the New Zealand priority scoring system - NZ score ${ }^{7}$ ), adjusting for evidence of co-morbidity and treatment preferences. We chose the NZ score not because it was well validated as a priority scoring instrument, but because it was the only one available.

We found:

- very little mismatch between the estimated need for and the amount of total hip replacement in the late 1990s (both around 45,000/annum);

- a much greater mismatch between the need for and provision of total knee replacements at the same time (estimated need around 55,000/annum compared with provision of around 30,000/annum);

- differences in the willingness to undergo surgery more people with a given level of disease were willing to have a hip replaced than a knee;

- important gender differences in the provision of care, women being less likely to have received drug therapy, been referred for specialist care or considered for joint replacement than men. These differences remained after adjustment of the data for co-morbidities and willingness to undergo surgery, and suggest that there may be important issues of sex discrimination in primary care.

Taken together these data emphasize the importance of the provision of hip and knee joint replacements to the NHS, but also indicate that there are significant problems to be addressed; it would appear that both the public and health care professionals need more help to ensure equitable access to, and provision of, joint replacements.

\section{The views and experiences of patients, professionals and the public}

The stakeholders involved in TJR include the patients, the doctors whom they first seek help from and who 
may refer them to the surgeons, and the surgeons themselves, as well as the general public who are taxed to provide the money for these interventions. We tried to gain a better understanding of the views of each of these groups about the provision of TJR for people with OA.

General practitioners: Both our theoretical and epidemiological work on access to TJR emphasized the importance of the 'gatekeeper' - the person who controls access to orthopaedic surgeons. In the UK this is largely the general practitioner (GP). Therefore, we thought it important to obtain data from GPs and other professionals that refer patients on what they thought about the indications and priorities for surgery. Within the 'EUROHIP' project we have undertaken a survey of the views of 304 orthopaedic surgeons and 314 referring physicians. ${ }^{18,19}$ Worrying differences in views both within and between groups emerged. One of the important findings within the data was the fact that, in general, the referring physicians had a higher threshold of disease severity for the consideration of TJR than the surgeons. This suggests that some people who the surgeon would consider appropriate for an operation are not being referred because the GP does not think they are severe enough.

As part of her PhD work, Caroline Sanders interviewed 11 GPs. $^{20}$ She found that they tended to explain the occurrence and progression of $\mathrm{OA}$ as a form of 'wear and tear' often associated with normal aging, a similar view to those of the people living with OA interviewed by Sanders. ${ }^{21,22}$ Although keen to harness the self-help capacities of their patients, GPs also seemed to make assumptions about what treatments specific groups of patients would want or not want, often assuming that older people might not want surgery. This is worrying, as expectations and assumptions about illness and disability in older age are known to influence treatment trajectories, and the views of the GPs involved in this study indicated that some patients might have had unmet needs for treatment. In relation to referral for surgery, the GPs wanted to be able to use scoring systems (such as the $\mathrm{NZ}$ score referred to above), to help them gain objective data on the need for referral.

Patients: Caroline Sanders, and another PhD student funded by the HSRC, Gillian Woolhead, also examined the views of patients on the indications and priorities for joint replacement. They found evidence for unmet need in the community, ${ }^{21,22}$ identifying three main barriers to access: people's own perceptions of need and risk leading to reluctance to seek help; negative experiences in primary care (such as being told that 'nothing can be done' about their arthritis); and previous experiences of treatment in secondary care resulting in undue worries about outcomes. These findings have recently been reinforced by work done by Jiri Chard for his HSRC-based PhD thesis, which highlighted the importance of negative views among health care professionals about OA and its treatment as a barrier for both patients and professionals to the provision of timely TJRs. ${ }^{23}$ Woolhead also explored what patients thought was happening in terms of the prioritization of individuals for a TJR, contrasting this with what they thought should happen, ${ }^{24}$ and found that patients, like the health care professionals, think that pain and disability should be the key criteria, but that they had additional issues that they thought the professionals should, but were not taking into account; these included the length of suffering, paid employment and the payment of National Insurance contributions, and caring for dependants. The patients that Woolhead interviewed thought that the doctors took too much notice of age, weight and excessive complaining by other patients. Woolhead also talked to some of her patients after they had had a total knee replacement to find out what they made of the experience and outcome. ${ }^{25}$ She reported that most of them struggled to make sense of what had happened and while publicly reporting good outcomes, many privately admitted that they still had a good deal of pain in the operated knee, with associated disability. Furthermore, many of those with ongoing pain blamed themselves, thinking that they must either have done too much or too little exercise in the immediate postoperative period. This work highlighted another problem experienced by patients undergoing a TJR in the UK: the lack of clear guidance on postoperative activity/exercise levels.

Surgeons: Nicola Hobbs is currently using patient vignettes to examine the factors that influence surgeons' prioritization for hip replacement surgery, asking them to rank patient vignettes that differed in level of pain severity, activity limitations, participation restrictions and patient motivation. Pain was more influential than either activity limitations or participation restrictions, but patient motivation was more influential than anything else in determining their hypothetical prioritization of patients for surgery. Next we will undertake in-depth interviews of orthopaedic surgeons, along with observational studies of consultations between surgeons and patients about the possibility of a primary TJR.

This body of work has highlighted several areas of concern, including a number of barriers to access, a mismatch within and between the views of the public, patients and health care professionals on the indications for a TJR, and patient prioritization. It seems likely that these problems result in serious inequities in provision in the UK. But the work has also suggested some possible solutions, such as working with the 'capacity to benefit' idea, as outlined further below.

\section{Measurement of outcomes in TJR}

In order to be able to accurately evaluate the outcomes of TJR and understand the processes involved, it is important to use appropriate measurement instruments. A measure that defines the construct of interest enhances compatibility, comparisons and understanding between studies. In order to change or manipulate something, it is necessary to understand how it operates. Hence, it is advantageous if a measure is assessing 
a construct that is part of a theoretical model. A major strand of the MOBILE programme, led by Marie Johnston and her colleagues Beth Pollard and Diane Dixon in Scotland, is designed to make more sense of the theory and practice of health status and outcomes measurement for people with OA undergoing a TJR. This work has primarily focused on self-report methods, but has also involved direct observation, proxy reports and electronic measurement of activity using accelerometers.

A review of existing commonly used measures to assess OA found wide variation in methodological development and little relationship to theoretical models. ${ }^{26}$ While patient self-reported measures had, in general, good methodological development, the review highlighted the relatively poor development of clinician-reported measures.

The International Classification of Functioning, Disability and Health $\left(\mathrm{ICF}^{27}\right)$ has become widely adopted as the theoretical framework of choice. The ICF is based upon a biopsychosocial model of functioning and has three main components: impairment (I); activity limitation (A); and participation restriction (P). In examining the relationships between $\mathrm{I}, \mathrm{A}$ and $\mathrm{P}$, it is important to establish that each measure is assessing only a single theoretical construct and not other constructs; otherwise, observed relationships between constructs may be misleading and interventions may be inappropriately targeted. Work by Beth Pollard has shown, based on classifications by expert judges, that existing OA measures contain a mixture of ICF constructs ${ }^{28,29}$ and are, therefore, unsuitable for investigations based on the ICF.

However, a pool of 'uncontaminated' items was identified by the expert judges; i.e. items that were measuring a single ICF construct. These 'uncontaminated' items formed the basis of new measures of the ICF components of impairment, activity limitation and participation restriction. The items were examined using responses from a geographical cohort of patients (JR-600 - see below) shortly before and 12 months after having a hip or knee replacement. Item analysis of the preoperative data was carried out combining item response theory and classical test theory methods, and led to the removal of items that did not fit. The new measures appear to provide a more accurate estimation of the ICF pathways than pre-existing measures, ${ }^{30}$ and they are currently undergoing further refinement and empirical testing. Additionally, the use of these measures of impairment, activity limitation and participation restriction, is currently being explored postoperatively.

Findings of relevance to the assessment of outcomes in TJR include:

- existing measures of outcome in OA and TJR assess a mixture of impairment, activity limitation and participation restriction and are, therefore, unsuitable for measuring ICF constructs;
- using the method of Discriminant Content Validity, ${ }^{28}$ it is possible to identify questionnaire items which are 'uncontaminated' by the other constructs and to develop new, pure measures;

- self-report, proxy report, observation and accelerometer assessments of disability are correlated, but do not show sufficient agreement to be used interchangeably; ${ }^{31}$

- all methods of measurement showed some correlation with negative mood, i.e. negative affectivity bias is not restricted to self-report; ${ }^{31,32}$

- in people who report activity limitations which do not accord with their underlying impairment, those reporting unexpectedly low activity levels give a more accurate representation of their true levels (measured by accelerometers) than those reporting unexpectedly high levels of activity (work with Derek Johnston and Julia Hay).

In collaboration with an international group of investigators, working under the auspices of the Osteoarthritis Research Society International (ORSI) and the Outcomes in Rheumatology Group (OMERACT), we have also been looking at the measurement of pain in OA. ${ }^{33}$ The main contributors to this from the MOBILE group have been Rachael Gooberman-Hill and her PhD student Fiona Mackichan. They carried out focus groups with patients with hip or knee OA of varying severity, ${ }^{34}$ and using the 'questerviews technique, ${ }^{35}$ (a methodological approach developed within the HSRC), sought their views on the questions used to assess pain impairment and resulting activity limitations within the WOMAC, the most commonly used outcome instrument in studies of OA. ${ }^{36}$ Findings of relevance to the assessment of pain included:

(1) pain is intermittent and variable, so the recording of severity on a pain measure will depend entirely on when the question is asked;

(2) pain elsewhere in the body is common and influences the experience of joint pain, so it is naïve to ask about pain in a single joint in isolation;

(3) pain and function cannot be separated as is attempted in most standard measures, as the amount of pain depends largely on what a person does;

(4) further to the above, adaptation and avoidance strategies are very widely used by patients and these modify the pain experience; and

(5) in addition to their 'usual' aching activity-related pain, most people with hip or knee OA experience spontaneous, short-lasting episodes of a more severe form of joint pain.

These findings, and other work from the wider OARSI/OMERACT group, have led to the development of a new instrument that takes account of 'usual' pain, as well as episodic attacks, avoids muddling pain with function and tries to assess pain distress 
as well as pain severity. The instrument is currently being validated.

Paul Dieppe and his PhD student Vikki Wylde are developing further work on pain in OA. The finding that many patients experience spontaneous attacks of pain, as well as other published work, suggests that central pain sensitization mechanisms (in the spinal cord or brain) may be as important as local pain driven from the joint. So we are now using quantitative sensory testing (QST) combined with detailed pain histories, to try to identify patients in whom pain sensitization might be of paramount importance. We hypothesize that such patients would respond relatively poorly to a TJR (Dieppe et al., submitted for publication). ${ }^{37}$

\section{Theory}

The measurement work has highlighted the lack of theory underpinning existing measurement instruments and understanding of the process of disablement. The use of measures that have a theoretical foundation should lead to more accurate evaluations of health outcome before and after TJR. In addition, we have worked to develop the ICF from a taxonomy of health outcomes to an explanatory model of disability. The ICF suggests a pathway from pain impairment to activity limitations to participation restriction. This pathway is represented in patients' spontaneous reports. Patients were asked what were the three most important things they hoped for from their joint replacement. Their responses were classified according to the main ICF components (impairment, activity limitation and participation restriction). The most common pattern of responses was that the patients' first hope related to impairment (e.g. 'I want to be pain free'), their second hope related to activity limitation (e.g. 'easier walking') and their third hope related to participation restriction (e.g. 'to be able to play golf'). Thus patients' comments appear to fit the ICF modelling approach.

However, we have additionally attempted to build on that model by incorporating psychological constructs. The activity limitations and participation restriction constructs in the ICF are forms of human behaviour and, therefore, invite the application of theories of behaviour and behaviour change to further our understanding of the processes that influence disability. We have incorporated models of behaviour, such as Social Cognitive Theory ${ }^{38}$ and the Theory of Planned Behaviour, ${ }^{39}$ into the ICF to develop an integrated model of disability ('disability as behaviour"40 (Figure 2). In this integrated model, psychological theory can be seen to operationalize the 'personal' factors construct in the ICF. Consequently, psychological factors mediate the relationship between impairment, activity limitations and participation restrictions.

We have employed structural equation modelling to test the ability of the integrated model to account for mobility disability in people awaiting TRJ using



Figure 2 Integrated model of disability: the theory of planned behaviour incorporated into the ICF. PBC, perceived behavioural control

a subset of patients from the JR-600 study. Diane Dixon has tested the integrated model.

Findings of relevance to understanding the relationships between pain impairment and walking disability in OA included:

- the new 'pure' measures give stronger evidence of the theoretical links between I, A and P than existing 'contaminated' measures;

- people awaiting TJR intuitively describe their goals for surgery in terms of the ICF model - i.e. predominantly putting I first, followed by A, followed by P;

- the ICF model accounts for $28 \%$ of the variance in walking limitations in patients with OA awaiting TJR;

- psychological theory accounts for $48 \%$ of the variance in walking limitations in patients with $\mathrm{OA}$ awaiting TJR;

- the integrated model (ICF plus psychological theory) accounts for $57 \%$ of the variance in walking limitations in patients awaiting TJR;

- personal control cognitions significantly mediated the relationship between impairment and activity limitations indicating that interventions that target such cognitions could contribute to optimal recovery;

- I, A and P have varying impacts on patient distress in different disabling conditions; ${ }^{41}$

- $\mathrm{N}$ of 1 methods can be used to test the ability of the integrated model to explain disability within a single individual (work by HSRC PhD student Francis Quinn).

The ICF also recognizes that 'environmental' factors influence the relationships between I, A and P. Future work with the integrated model will incorporate measures of the 'environment', in the form of socioeconomic status and educational attainment, with the aim of improving the explanatory power of the integrated model.

\section{Cohort studies and the determinants of good and bad outcomes after TJR}

One way of finding out who will benefit most from an intervention like a TJR is to undertake a clinical trial. A waiting list trial, of the sort used to investigate 
cataract surgery, ${ }^{42}$ is one option. As mentioned in the introduction, when this programme of work began, we had planned to do such trials, but we were unable to obtain ethical committee approval, even to undertake pilot studies. The main reason given was that everyone 'knew' that TJRs were effective and trials were not necessary. So we have fallen back on cohort studies to try to ascertain the determinants of good and bad outcomes.

We began by looking at some existing databases, such as the Avon Knee Registry, ${ }^{43}$ but these proved to be of little value, because of the paucity of relevant baseline data. So, we have developed two new cohorts of our own:

(1) The 'JR-600' cohort: As the name implies, this is a cohort of 600 people with OA, undergoing a primary hip or knee replacement. It is based at Dundee in Scotland, and Beth Pollard, Diane Dixon, Marie Johnston and David Rowley are the primary investigators. As mentioned above, it is being used to investigate our new approach to outcome assessment and to understanding activity limitations within an integrated theoretical model. The data collected contains a wealth of information that should help us to uncover some of the factors that determine good or bad outcomes. In particular, we have collected a large amount of information on the psychological status of the patients preoperatively as suggested by our integrated theoretical approach;

(2) The 'EUROHIP' cohort: The 'EUROHIP' consortium is led by Paul Dieppe, Wilfred von Eiff (Munster) and Wolfhart Puhl (Ulm). It includes 20 orthopaedic centres in 12 different countries, and has been investigating the indications, process and outcomes of primary total hip replacements. ${ }^{18,19}$ Paul Dieppe is the lead investigator of a cohort study being undertaken by the group. Data have been collected from 1520 patients, most of whom have been adequately assessed both preoperatively and one year later. The data include WOMAC and EQ5D at both time points, as well as extensive preoperative data on demography, co-morbidities and social status. An initial analysis of the baseline data has emphasized the huge variation in the severity of the disease at the preoperative assessment. Educational status was one of the strong predictors of disease severity; those who were more educated having significantly less severe disease at the time of surgery than those with lower educational attainments. We will be undertaking analyses of the determinants of outcome in this cohort soon.

In addition to the clinical data available in these cohorts, we have radiographs, which are being assessed in a uniform way by two assessors (Paul Dieppe and Sue Williams) to help to relate structural damage to health status and outcomes.

\section{Bringing it all together, where this work should go next}

The MOBILE programme has been the vehicle for a large body of work on TJR for OA of the hip and knee in the UK. The work so far has highlighted four main areas of concern:

(1) indications for surgery, prioritization and access;

(2) outcome assessment;

(3) how impairment combines with psychological factors in the development of activity limitations;

(4) perioperative care.

\section{Indications for surgery, prioritization and access}

It is clear that there is confusion about who should be considered for a TJR, with major differences in views both within and between different groups of professionals, and with patients and the public suggesting approaches that are quite different from those of their doctors. Furthermore, we have found a lot of evidence for inequities in provision in England, and likely inequalities - older people and women seem to be particularly likely to be treated 'unequally'. The framework that we started out with can now be improved upon with data to support the existence of key barriers to access to surgery (Figure 1).

How can the situation be improved? GPs are asking for guidance on who they should refer to surgeons, and are increasingly being asked to use crude scoring systems such as the New Zealand priority score. ${ }^{7}$ But such scores do not take account of the issues that patients and the public think should be used when trying to make such decisions (such as carer roles). We are taking two different approaches to this:

(1) Appropriateness criteria: Appropriateness criteria have been developed for a number of surgical interventions, generally using the approach developed by the RAND organization. ${ }^{44}$ There have been attempts to do this for joint replacement, ${ }^{45}$ and the EUROHIP consortium has recently produced new criteria that take account of a wider spectrum of factors and are, we believe, an improvement (Floren et al., submitted for publication). A way forward would be to adapt these criteria locally with the help of patients as well as professionals. We are recommending the use of the data that we have, including both our appropriateness criteria, and the framework mentioned below, by local consortia of patients and professionals to aid shared understanding between the 'gatekeepers', the surgeons, and health care managers on referral and prioritization for TJR;

(2) A framework based on 'capacity to benefit': The views of the public and of patients undergoing TJR indicate that to them the answer is simple - 'those people who are going to gain the most from the intervention are the ones that you should prioritize for 
surgery'. Many of them seemed rather surprised that we did not know who those people were! Clearly our cohort studies, in addition to current work in many other centres, should help to understand the determinants of relatively good or poor outcomes after surgery, and hence aid prioritization by 'capacity to benefit' - a construct that involves the value of alternatives and the risks from surgical intervention as well as the likely reduction in pain and disability. We are working on populating and testing a framework for this construct, which might be of value to both patients and professionals (Figure 3). ${ }^{46}$

\section{Outcome assessment}

The majority of published work on the 'success or failure' of a TJR is about technical prosthesis failure i.e. loosening or infection. Although things have changed for the better over the last decade, this is still the dominant paradigm in orthopaedics, in spite of the fact that patient-related outcomes do not appear to have much relationship to the technical outcome, at least in the short term. We believe that a much more sophisticated approach to outcome assessment is needed to allow us to understand what is going on and how to improve things for the patients. We hope that our new measures of pain, impairment, activity limitations and participation restrictions (the ICF domains), mentioned above, will help this, and we intend to promote their use in rheumatology and orthopaedics. In addition, we are applying this approach to trials, re-analysing the effects of interventions to see if they differentially affect I, A and P. Our new measures cannot be used for utility assessment, and we have not looked into the issue of QUALs and the costeffectiveness of TJR; however, work within the linked
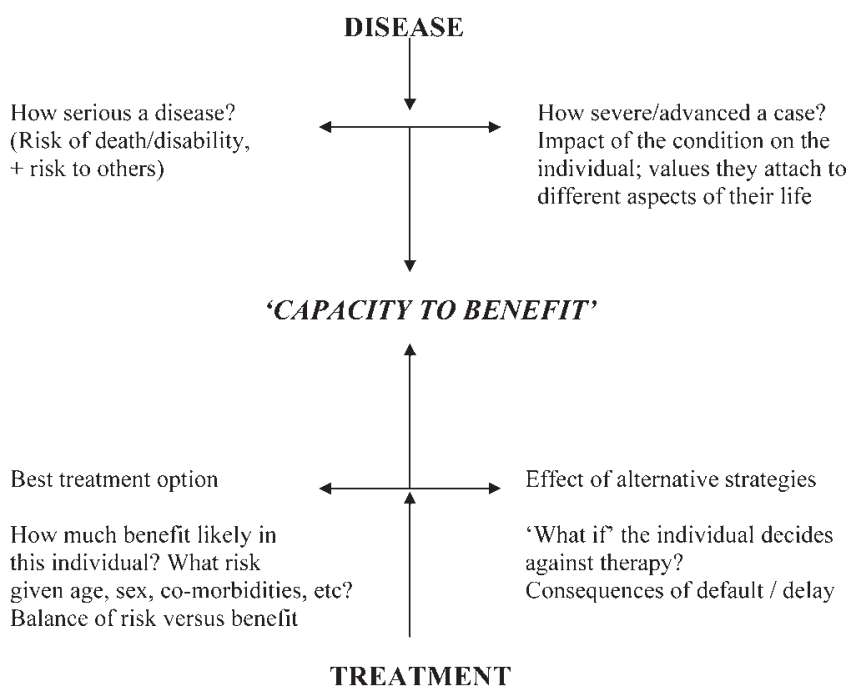

Figure 3 Preliminary conceptual model of a framework for the 'capacity to benefit' from a total joint replacement
'ICEPOP' programme is developing that area, as outlined by Coast et al. in this volume. ${ }^{47}$

\section{How impairment combines with psychological factors in the development of activity limitations}

The work of Marie Johnston and her colleagues in Scotland is leading to new ways of thinking about what factors need to be considered to improve outcomes.

The ICF suggests relationships between impairment and disability. The main pathways of the ICF model have been explored using the newly developed ICF measures (see above). Using data from the JR-600 cohort, Marie Johnston, Beth Pollard and Diane Dixon have shown that there are significant pathways between impairment and activity limitation and between activity limitation and participation restriction, but a non-significant relationship between impairment and participation restriction. These pathways appeared to be consistent with the way patients view their condition. Additionally, they suggest that interventions aimed at reducing impairment should also reduce activity limitation and participation restriction, whereas an intervention aimed at improving participation may not impact on the two other components of the ICF.

We are currently testing the ability of the integrated model of disability to account for recovery from TJR in patients from JR-600 and in n-of-1 studies. These analyses should also identify cognitions that predict recovery of function. The JR-600 study should demonstrate the ability of the integrated model to account for differences between individuals within a group, whereas n-of-1 studies should establish the ability of the model to account for change within a single individual. The concomitant use of these two methods should, therefore, go some way to addressing the clinicians' problem of applying outcomes generated from groups of individuals in trials to specific patients.

The aim of this work is to promote optimal outcome using interventions designed to impact on the cognitions that predict outcomes and thereby enhance recovery, and to reduce activity limitations independently of the need to reduce impairment. We are planning studies that go through the stages of intervention development, based on the integrated model, and subsequent evaluation in trials, for people in the community with OA and also prior to TJR .

\section{Perioperative care}

Our work has highlighted a large number of concerns about the process of TJR and patients' experiences in hospitals. In addition to the evidence for variations in different regions, apparent in the HES data, our interviews with patients indicate that pain control is poor and that they are given little or no advice or help with postoperative activity. As a result of this work, we have decided that perioperative care, including better 
patient-centred approaches to preoperative preparation, perioperative pain control and postoperative rehabilitation need to be a future priority.

We are currently undertaking literature searches concerning the delivery of care related to TJR, and pre- and postoperative pain control. There is little good trial evidence to help professionals understand what they should be doing, and the EUROHIP project has shown that there are massive differences in approach in the different participating countries and centres.

We believe that the way forward here is trials. We are planning trials to aid pain control perioperatively, to improve cognitions, and to improve general health and musculoskeletal function perioperatively. We have, in collaboration with Ashley Blom and Ian Learmonth in the Avon Orthopaedic Centre in Bristol, obtained NIHR programme grant funding to take this work forward.

\section{Conclusions}

It is 45 years since Charnley published his paper 'Arthroplasty of the hip: a new operation' in the Lancet. ${ }^{1}$ The operation works well for most people. But we still do not know who is likely to benefit most, and in whom it will fail (clinically), or why. Until recently, very little consideration was given to how to assess outcomes or how they could be improved by optimizing perioperative care. We believe that the MOBILE programme has provided data that give clear indications as to the ways forward to improve the situation, at least in the UK.

The challenge now, as ever with multidisciplinary HSR, is how to implement our findings for maximum patient benefit. In that regard, we need the help of policy-makers and managers within the NHS. It is clear to us that although TJR is an important, effective and cost-effective procedure, patient experiences and outcomes could be improved, and inequalities reduced if we:

(1) made sure that joint replacements were only carried out in high volume teaching units which standardize their procedures;

(2) developed local consortia of health care professionals from primary and secondary care, along with patients who have had or need to have a TJR, to develop local appropriateness criteria for patients thought to be in need of a TJR, that could be 'owned' by both local GPs and orthopaedic surgeons;

(3) developed evidence-informed consensus criteria for standardized programmes of perioperative care and rehabilitation that can be investigated through clinical trials.

\section{Acknowledgements}

We are grateful to the MRC for funding the majority of this work, and to the Arthritis Research Campaign that funded part of it, as well as to the support of everyone in both the MRC HSRC and the EUROHIP consortium. We owe a special debt of gratitude to the members of the MOBILE team not listed as authors of this paper, in particular Salma Ayis, Rachael Gooberman-Hill and Andrew Beswick, to our orthopaedic colleagues in Dundee and Bristol who have supported our work so generously, and to all the PhD students and others who have contributed to this work.

\section{References}

1 Charnley J. Arthroplasty of the hip. A new operation. Lancet 1961;1:1129-32

2 National Institute for Health and Clinical Excellence. Osteoarthritis: the care and management of osteoarthritis in adults. London: NICE, 2008. See http://www.nice.org.uk

3 Insall JN, Dorr LD, Scott RD, Scott WN. Rationale of the Knee Society clinical rating system. Clin Orthop Relat Res 1989;248:13-14

4 Harris WH. Traumatic arthritis of the hip after dislocation and acetabular fractures: treatment by mold arthroplasty. An end-result study using a new method of result evaluation. J Bone Joint Surg Am 1969;51:737-55

5 NIH consensus conference: Total hip replacement. NIH Consensus Development Panel on Total Hip Replacement. JAMA 1995;273:1950-6

6 Naylor CD, Williams JI. Primary hip and knee replacement surgery: Ontario criteria for case selection and surgical priority. Qual Health Care 1996;5:20-30

7 Hadorn DC, Holmes AC. The New Zealand priority criteria project. Part 1: overview. BMJ 1997;314:131-4

8 Freund DA, Katz BP, Callahan CM. Patient outcomes research teams: examples from a study on knee replacement. Ann N Y Acad Sci 1993;703:86-94; discussion $94-5$

9 Havelin LI. The Norwegian Joint Registry. Bull Hosp Jt Dis 1999;58:139-47

10 Malchau H, Herberts P, Eisler T, Garellick G, Söderman P. The Swedish Total Hip Replacement Register. J Bone Joint Surg Am 2002;84-A (Suppl. 2):2-20

11 HSRC strategy presented to MRC in 1998

12 Dieppe P, Basler H-D, Chard J, et al. Knee replacement surgery for osteoarthritis: effectiveness, practice variations, indications and possible determinants of utilization. Rheumatology (Oxford) 1999;38:73-83

13 Dixon T, Shaw M, Dieppe PA. Analysis of regional variation in hip and knee joint replacement rates in England using Hospital Episodes Statistics. Public Health 2006;120:83-90

14 Dixon T, Shaw M, Ebrahim S, Dieppe P. Trends in hip and knee joint replacement: socioeconomic inequalities and projections of need. Ann Rheum Dis 2004;63:825-30

15 Judge A, Chard J, Learmonth I, Dieppe P. The effects of surgical volumes and training centre status on outcomes following total joint replacement: analysis of the Hospital Episode Statistics for England. J Public Health (Oxf) 2006; 28:116-24

16 Frankel S, Eachus J, Pearson N, et al. Population requirement for primary hip-replacement surgery: a crosssectional study. Lancet 1999;353:1304-9

17 Juni P. Population requirement for primary knee replacement surgery: a cross-sectional study. Rheumatology (Oxford) 2003; 42:516-21

18 Dreinhöfer KE, Dieppe P, Stürmer T, et al. Indications for total hip replacement: comparison of assessments of orthopaedic surgeons and referring physicians. Ann Rheum Dis 2006;65:1346-50

19 Stürmer T, Dreinhöfer K, Gröber-Grätz D, et al. Variations within, and differences between the views of orthopaedic surgeons and referring physicians on the determinants of outcome after total hip replacement. J Bone Joint Surg Am 2005;87:1416-19

20 Sanders C, Donovan JL, Dieppe PA. Unmet need for joint replacement: a qualitative investigation of barriers to 
treatment among individuals with severe pain and disability of the hip and knee. Rheumatology (Oxford) 2004;43:353-7

21 Sanders C. The significance and consequences of joint disease in older age. PhD thesis. Bristol: University of Bristol, 2004

22 Sanders C, Donovan J, Dieppe P. The significance and consequences of having painful and disabled joints in older age: co-existing accounts of normal and disrupted biographies. Sociol Health Illn 2002;24:227-53

23 Chard J, Dickson J, Tallon D, Dieppe P. A comparison of the views of rheumatologists, general practitioners and patients on the treatment of osteoarthritis. Rheumatology (Oxford) 2002;41:1208-10

24 Woolhead GM, Donovan JL, Chard JA, Dieppe PA. Who should have priority for a knee joint replacement? Rheumatology (Oxford) 2002;41:390-4

25 Woolhead G, Donovan J, Dieppe P. Outcomes of total knee replacement: a qualitative study. Rheumatology (Oxford) 2005; 44:1032-7

26 Pollard B, Johnston M, Dixon D. Theoretical framework and methodological development of common subjective health outcome measures in osteoarthritis: a critical review. Health Qual Life Outcomes 2007;5:14-23

27 World Health Organization. International Classification of Functioning, Disability and Health. Geneva: WHO, 2001

28 Pollard B, Johnston M, Dieppe P. What do osteoarthritis health outcome instruments measure? Impairment, activity limitation, or participation restriction? J Rheumatol 2006;33:757-63

29 Pollard B, Johnston M. The assessment of disability associated with osteoarthritis. Curr Opin Rheumatol 2006;18:531-6

30 Pollard B. Measuring subjective health outcomes: methodological and theoretical considerations. PhD thesis. St Andrews: University of St Andrews, 2007

31 Powell R. Psychological aspects of activities limitations. PhD thesis. Aberdeen: University of Aberdeen, 2006

32 Powell R, Johnston M, Johnston DW. Assessing walking limitations in stroke survivors: are self-reports and proxy reports inter changeable? Rehabil Psychol 2007;52:177-83

33 Singh JA, Solomon DH, Dougados M, et al. Contributions of OMERACT to rheumatic disease research. Arthritis Rheum 2007;57:186

34 Gooberman-Hill R. Assessing chronic joint pain: lessons from a focus group study. Arthritis Rheum 2007;57:666-71
35 Adamson J, Gooberman-Hill R, Woolhead G, Donovan J. 'Questerviews': using questionnaires in qualitative interviews as a method of integrating qualitative and quantitative health services research. J Health Services Res Policy 2004; 9:139-45

36 Bellamy N, Buchanan WW, Goldsmith CH, Campbell J, Stitt LW. Validation study of WOMAC: a health status instrument for measuring clinically important patient relevant outcomes to antirheumatic drug therapy in patients with osteoarthritis of the hip or knee. J Rheumatol 1988;15:1833-40

37 Wylde V, Dieppe P, Hewlett S, Learmonth ID. Total knee replacement: is it really an effective procedure for all? Knee 2007; 14:417-23

38 Bandura A. Self-Efficacy: The Exercise of Control. New York, NY: WH Freeman, 1997

39 Ajzen I. The theory of planned behaviour. Organ Behav Hum Dec Process 1991;50:179-211

40 Dixon D. Conceptual and measurement models of disability. Unpublished doctoral dissertation. Aberdeen: University of Aberdeen, 2006

41 Schroeder C, Johnston M, Teunissen L, et al. Perceived control is a concurrent predictor of activity limitations in patients with chronic idiopathic axonal polyneuropathy. Arch Phys Med Rehabil 2007;88:63-9

42 Laidlaw DA, Harrad RA, Hopper CD, et al. Randomised trial of effectiveness of second eye cataract surgery. Lancet 1998;352:925-9

43 Kennedy LG, Newman JH, Ackroyd C, Dieppe PA. When should we do knee replacements? Knee 2003;10:161-6

44 Brook RH. Appropriateness: the next frontier. BMJ 1994; 308:218-19

45 Quintana JM, Escobar A, Arostegui I, et al. Health-related quality of life and appropriateness of knee or hip joint replacement. Arch Intern Med 2006;166:220-6

46 Dieppe P, Davies L, Donovan J, Learmonth I, Sanders C, Woolhead J. Indications for total joint replacement in osteoarthritis: a new framework. Reumatismo 2005;57 (Numero Speciale 1): $168-70$

47 Coast J, Flynn T, Sutton E, et al. Investigation Choice Experiments for Preferences of Older People (ICEPOP): evaluative spaces in health economics. J Health Serv Res Policy 2008;13 (Suppl. 3):31-7 